Wojciech Bałus

\title{
MOCAK und der Mythos der Modernität in Krakau
}

2004 initiierte das polnische Kulturministerium das Programm „Zeichen der Zeit“. Dessen Ziel war es unter anderem, ein Netzwerk von Museen und interdisziplinären Zentren für Kultur der Gegenwart zu bilden, die Kulturwerke unserer Zeit sammeln, aufbewahren und zugänglich machen sollten. ${ }^{1}$ Ein Jahr später wurde die Investitionsaufgabe „Einrichtung des Museums für Zeitgenössische Kunst“ im Stadtbudget Krakaus berücksichtigt. ${ }^{2}$ Das Gebäude der neuen Institution, des MOCAK (Museum of Contemporary Art Kraków), entstand in Folge eines internationalen Wettbewerbs, der 2007 entschieden wurde. Der Siegerentwurf stammte von den italienischen Architekten Claudio Nardi und Leonardo Prioli. Das Museumsgebäude wurde - allerdings noch als Rohbau - am 16. November 2010 feierlich eröffnet, mitten im Wahlkampf vor den Selbstverwaltungswahlen. ${ }^{3}$ Die ersten Ausstellungen konnten erst ein halbes Jahr später, ab 19. Mai 2011 besucht werden (damals fand die abermalige Eröffnung statt).

Das MOCAK-Gebäude wurde auf dem Gebiet von Zabłocie, am rechten Weichselufer errichtet. $\pi$ Abb. 1 Das historische Krakau entwickelte sich bis zum 19. Jahrhundert am linken Ufer des Flussbettes; nur das Stadtviertel Kazimierz mit dem jüdischen Viertel war zwischen dem Hauptflussbett und dem Arm der Alten Weichsel, die in der 2. Hälfte des 19. Jahrhunderts zugeschüttet wurde, gelegen. Bei der ersten Teilung Polens 1772 wurde die Grenze zu Österreich entlang der Weichsel gezogen. Bald darauf entstand - gegenüber Krakau - die österreichische Stadt Podgórze (1784). ${ }^{4}$ Zabłocie, ein Dorf mit Wurzeln im Mittelalter, wurde Bestandteil dieses neuen städtebaulichen Organismus. ${ }^{5}$

Ein besonderer Status kam Krakau im 19. Jahrhundert zu. Als alte Hauptstadt Polens wurde es zu einem patriotischen Wallfahrtsort für die Bevölkerung des ganzen Landes. Man kam nach Krakau, um die Denkmäler der nationalen Vergangenheit zu besichtigen: die Wawel-Kathedrale (Begräbnisstätte der meisten historischen Herrscher Polens), das Königsschloss, die Marienkirche mit dem Veit-Stoß-Altar und andere historische Bauten und Kunstwerke. Die Stadt wurde bald als „Reliquiar nationalen Erinnerungsgutes“ und „geistige Hauptstadt Polens“

1 Narodowy Program Kultury Znaki Czasu na lata 2004-2013, http://bip.mkidn.gov.pl/media/docs/ NPK_Znaki_Czasu.pdf (Stand: 20.02.2012).

2 Utworzenie Muzeum Sztuki Współczesnej w Krakowie, Dokument im Archiv von MOCAK.

3 Dawid Hajok, Tomasz Handzlik: Stare i nowe w muzeum, Gazeta Wyborcza (dodatek krakowski), 17.11.2010, S. 3.

4 Rudolf A. Mark: Galizien unter österreichischen Herrschaft. Verwaltung - Kirche - Bevölkerung, Marburg 1994, S. 1, 4.

5 Michał Wiśniewski: Zabłocie - a perfect example of revitalisation?, Herito 4, 2011, S. 60. 


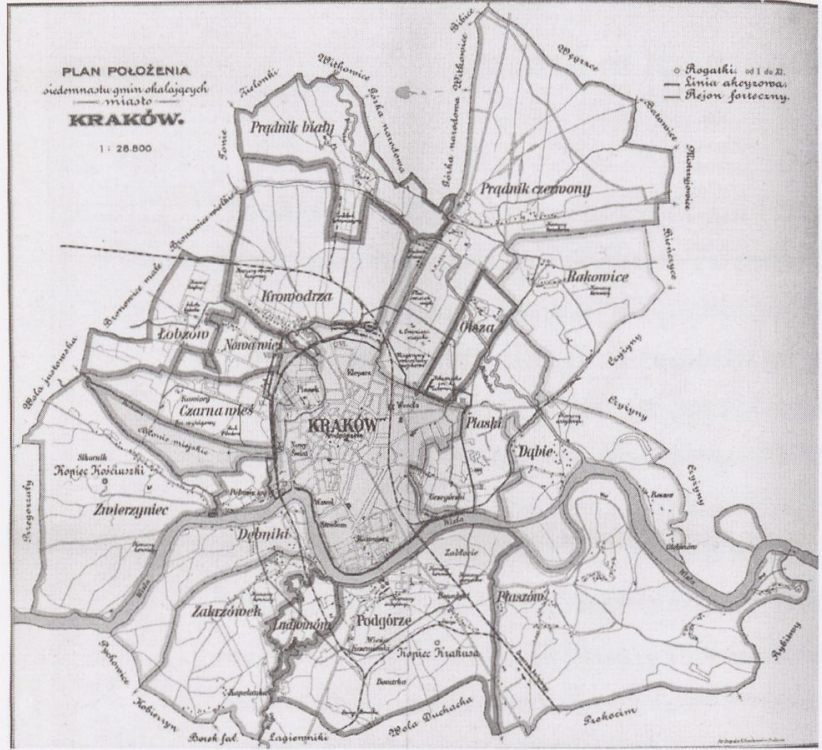

1: Das große Krakau, Stadtplan von 1910,

Zabłocie rechts unten zwischen Podgórze und Płaszów. apostrophiert. Dank der Autonomie, die Galizien 1866 erlangte, konnte sich in Krakau auch die polnische Kultur ziemlich frei entwickeln: Es existierte bereits die repolonisierte Jagiellonen-Universität (gestiftet 1364), gegründet wurden die Akademie der Wissenschaften und das erste Nationalmuseum. Die Stadt wurde auch zum Zufluchtsort für zahlreiche von Germanisierungsund Russifizierungsmaßnahmen betroffene Flüchtlinge aus dem russischen und dem preußischen Teilungsgebiet. ${ }^{6}$

In der 2. Hälfte des 19. Jahrhunderts stützte sich die Entwicklung Krakaus hauptsächlich auf sein immaterielles Vermögen. Entscheidend für den Aufschwung der Stadt war ihre symbolische Funktion. ${ }^{7}$ Eine verspätete Modernisierung und Industrialisierung setzten erst zu Ende des Jahrhunderts ein. Die Standorte der meisten Industriebetriebe befanden sich aber außerhalb der Stadt - in dem immer noch selbstständigen Podgórze, vor allem auf dem Gebiet von Zabłocie, unweit der Eisenbahnlinie. ${ }^{8}$ Hoffnungen auf eine weitere Entwicklung Krakaus wurden am Anfang des 20. Jahrhunderts durch österreichische Pläne zum Bau eines Donau-Oder-Weichsel-Dnjestr-Kanals entfacht, in denen die einstige Hauptstadt eine nicht unwesentliche Rolle als großer Binnenhafen spielen sollte. ${ }^{9}$ Die Umsetzung der Pläne wurde zwar durch den Ersten Weltkrieg durchkreuzt, doch die Idee des Kanals ließ die Bemühungen um eine Erweiterung der Stadt ausreifen. 1909 gelang es, die entsprechenden Verträge mit einem Dutzend Nachbargemeinden zu unterzeichnen, allerdings ohne Podgórze, das am längsten - bis 1915 - der

6 Wojciech Bałus: Krakau zwischen Traditionen und Wegen in die Moderne. Zur Geschichte der Architektur und der öffentlichen Grünanlagen im 19. Jahrhundert, Stuttgart 2003, S. 23-32.

7 Jacek Purchla: Krakau unter österreichischen Herrschaft 1846-1918. Faktoren seiner Entwicklung, Wien/Köln/Weimer 1993.

8 Jacek Purchla: Kraków i Lwów wobec nowoczesności, w: Kraków i Galicja wobec przemian cywilizacyjnych (1866-1914), Kraków 2011, S. 232.

9 Jacek Purchla: Der Anfang war auch schon das Ende, Austria Today 1992, D2, S. 29-30. 
Eingemeindung widerstand. ${ }^{10}$ Als Symbol dieses Widerstandes und einer immer wieder betonten Souveränität kann der Turm der neugotischen Pfarrkirche (gebaut 1903-1909) gedeutet werden, der die Form des höheren Turmes der gotischen Marienkirche, eines der markantesten Wahrzeichen Krakaus, wiederholte. ${ }^{11}$

In der Zwischenkriegszeit wahrte Zabłocie seinen industriellen Charakter. Unter den vielen damals funktionierenden Betrieben war auch die Email- und Blechwarenfabrik Rekord, gegründet drei Jahre vor dem Ausbruch des Zweiten Weltkriegs von den jüdischen Unternehmern Izrael Kohn, Wolf Luzer Glajtman und Michał Gutman. Nachdem das Werk im Juni 1939 Konkurs angemeldet hatte, wurde es im November im gleichen Jahr, d.h. schon nach der Besetzung Polens durch Hitlerdeutschland, von Oskar Schindler übernommen. Er erweiterte die Fabrik, indem er neue Produktionshallen und das Verwaltungsgebäude in der Lipowa-Straße 4 (gebaut 1941-1942) errichten ließ. ${ }^{12}$ Nachdem sich der deutsche Unternehmer „bei dem sadistischen Kommandanten des KZ Plaszow Amon Göth die Möglichkeit erbeten hatte, ein Unterlager an diesem Ort einzurichten, konnte er hierher einen Teil der Gefangenen aus dem KZ-Mutterlager verlegen, wodurch er ihnen das Leben rettete. Es waren hauptsächlich Gefangene jüdischer Herkunft, wobei zu bemerken ist, dass nicht nur Schindler ihnen das Leben rettete. Ähnlich handelten die deutschen Eigentümer der Firmen Madritsch und Optima, sowie der Pole, Ingenieur Chmielewski, der die Aufsicht über die für die Deutschen wichtige Barracken-Fabrik hatte“ ${ }^{13}$ In Podgórze, nicht weit von Zabłocie, befand sich auch das Ghetto (in den Jahren 1941-1943).

In der Nachkriegszeit blieb Zabłocie weiterhin ein Industrieviertel. Die kommunistischen Behörden strebten eine Veränderung der sozialen Struktur des „bürgerlichen“ und „reaktionären“ Krakaus an - aus diesem Grund legten sie groBen Wert auf eine weitere Industrialisierung und massive Zufuhr der Arbeiter, die einen starken Kontrapunkt zu den bisherigen intellektuellen, künstlerischen und kaufmännischen Eliten der Stadt bilden sollten. ${ }^{14}$ Neben dem prestigeträchtigen

10 Purchla: Kraków i Lwów (wie Anm. 8), S. 231-232; Hanna Kozińska-Witt: Krakau in Warschaus langem Schatten. Konkurrenzkämpfe in der polnischen Städtelandschaft 1900-1939, Stuttgart 2008, S. 97-103.

11 Bałus (s. Anm. 6), S. 43-44.

12 Jacek Salwiński: Wokół ulicy Lipowej, Kraków 2011, S. 53-54.

13 Maciej Miezian: Najsłynniejsza fabryka. In: Joanna Targoń, Ryszard Kozik (Hg.): Muzeum Sztuki Współczesnej w Krakowie, Dodatek do Gazety Wyborczej, 23.10.2010, S. 11.

14 Jacek Purchla: Miasto niepokorne. Znaczenie okresu 1945-1956 dla rozwoju Krakowa po drugiej wojnie światowej, In: Ders.: Kraków - prowincja czy metropolia?, Kraków 1996, S. 134-135. 
Bau eines riesigen Eisenhütten-Kombinats samt einer neuen Stadt (Nowa Huta) wurden auch andere Betriebe ausgebaut und gegründet. In Zabłocie entstanden die Kosmetikfabrik Miraculum und die Telekommunikationsgeräte-Fabrik Telpod. ${ }^{15}$

Nach dem Untergang des Kommunismus ging auch die Industrie in Krakau bald zugrunde. Die Fabriken in Zabłocie wurden ebenfalls geschlossen. Eine verstärkte Degradierung des Viertels setzte ein. Erst am Anfang dieses Jahrhunderts zeichnete sich eine positive Wende ab. Einerseits ermutigte der enorm erfolgreiche Film Schindlers Liste von Steven Spielberg viele Touristen, Zabłocie zu besuchen. Nebenbei sei erwähnt, dass Krakau sich einer zunehmenden Popularität als Reiseziel erfreut: 2008 waren es über 7,5 Millionen Gäste! Andererseits begann 2004 der Prozess einer Gentrifizierung des Stadtviertels. In der Nähe von Zabłocie war schon 2001 eine Autobrücke gebaut worden, was zur besseren VerkehrserschlieBung dieses Gebiets beigetragen hatte. Die alten Industriebauten wurden immer häufiger zu Lofts umfunktioniert (z. B. die Getreidemühle) und ein Teil der alten Industriebausubstanz wurde abgetragen und durch Apartmenthäuser ersetzt (z. B.: Garden Residence). ${ }^{16}$ Auch öffentliche Einrichtungen kamen hinzu: das Gebiet der alten Kaserne wurde zum Standort der Krakauer Andrzej-Frycz-ModrzewskiAkademie, einer Hochschule mit universitären und künstlerischen Studiengängen.

2004 übernahm die Stadtgemeinde Krakau die Fabrik Schindlers als Ausgleich für Schulden. Es wurde dabei beabsichtigt, in dem Gebäude ein Museum unterzubringen. Anfangs plädierte man für eine Gedächtnisstätte, die mit der Vergangenheit der Fabrik und des Stadtteils hätte verknüpft werden sollen. ${ }^{17}$ Es gab auch Vorschläge, das neue Museum der Konspirationsorganisation Żegota zu widmen, die der jüdischen Bevölkerung im Zweiten Weltkrieg half. ${ }^{18}$ Die endgültige Entscheidung des Stadtpräsidenten Jacek Majchrowski, der die Fabrik Schindlers als Standort des Museums für Gegenwartskunst auswählte, sorgte für Proteste. Krakauer Stadträte der konservativen Partei von Jarosław Kaczyński Recht und Gerechtigkeit waren der Meinung, das Gebiet der Fabrik sei kein angemessener Ort, „einige zeitgenössische Kunstwerke“ zu zeigen, und schlugen eine andere Lösung vor: die Schaffung des Museums der Gerechten unter den Völkern, wofür

18 Muzeum Żegoty w Fabryce Schindlera, Gazeta Wyborcza (dodatek krakowski), 7.1.2005. 
sie sogar eine Unterstützung vonseiten Yad Vashems erhalten hatten. ${ }^{19}$ Die Stadtbehörden gingen einen Kompromiss ein - in dem erhaltenen Verwaltungsgebäude entstand eine Niederlassung des Historischen Museums der Stadt Krakau, die die Dauerausstellung Krakau - die Zeit der Besatzung 1939-1945 präsentiert, während das MOCAK-Gebäude, bei dem man spärliche Reste der historischen Architektur wiederverwenden konnte, anstelle der Fabrikhallen errichtet wurde.

Die Wahl von Zabłocie als Standort des Museums für Gegenwärtige Kunst war also eine Entscheidung der Stadt und ein klarer Ausdruck der von Krakau verfolgten Politik. In diesem Kontext verwundert es nicht, dass das Bauvorhaben vom Stadtpräsidenten als Argument im Wahlkampf verwendet wurde. Zugleich stellte Jacek Majchrowski bei der abermaligen und endgültigen Eröffnung des MOCAK, schon nach seiner Wiederwahl 2010, fest, das Museum sei „ein wesentliches Element der Revitalisierung der postindustrialen Gebiete in Zabłocie “ ${ }^{20}$ Seine Stadtpolitik habe somit eine Unterstützung für die Wiederbelebungspläne dieses vernachlässigten Viertels angesteuert.

Doch damit erschöpft sich die Frage des Standortes nicht. Dass einige öffentliche Einrichtungen am rechten Weichselufer lokalisiert wurden, jenseits des Bahndamms, der unausweichlich ein Zugangshindernis von der Seite der zentralen Teile des Stadtviertels darstellt, drängt die Frage auf, wie das politische Spiel auch in der symbolischen Topografie Krakaus ausgetragen wird. Und dies umso mehr, als die meisten Brücken, darunter auch die zuletzt in Betrieb genommene Fußgängerbrücke, nicht Zabłocie, sondern die weiter westlich gelegenen Teile von Podgórze mit dem Stadtviertel Kazimierz (und dadurch mit der Altstadt jenseits der Weichsel) verbinden.

In seinem Kommentar beschränkte sich Stadtpräsident Majchrowski allerdings nicht nur darauf, die Rolle der neuen Museen bei der Revitalisierung des Stadtviertels zu betonen. Er bemerkte auch, dass Zabłocie dank dieser Institutionen „langsam zu einem neuen Zentrum des kulturellen und gesellschaftlichen Lebens Krakaus wird“. ${ }^{21}$ Die Direktorin des MOCAK, Maria Anna Potocka, konstatierte hingegen bei der ersten Eröffnung des Gebäudes: „Krakau hat zu Unrecht den Ruf einer Stadt

19 Magdalena Kursa: Yad Vashem popiera Muzeum Sprawiedliwych, Gazeta Wyborcza (dodatek krakowski), 19.6.2007.

20 Jacek Majchrowski: Nowe centrum, In: Joanna Targoń, Ryszard Kozik (Hg.): Muzeum Sztuki Współczesnej w Krakowie, Dodatek do Gazety Wyborczej, 14.5.2011, S. 3.

21 Majchrowski (s. Anm. 20), S. 3. 


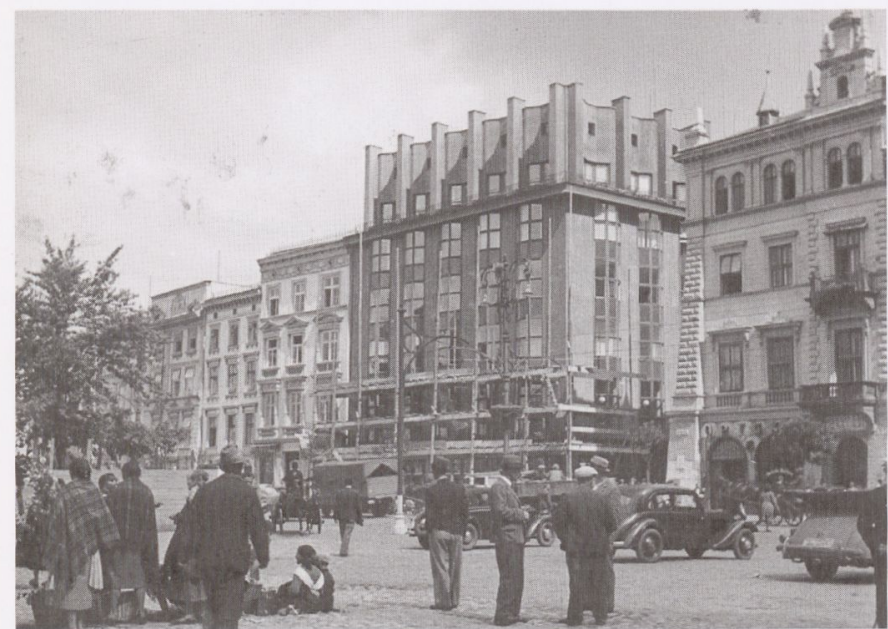

2: Krakau, Marktplatz mit dem Feniks-Gebäude in der letzten Phase des Baus, 1932 (Foto Archiwum Pałstwowe Kraków). gehabt, die sich im Sumpf der Geschichte wälzt, jetzt wird es sich ändern." 22 Seine Meinung äußerte damals auch Filip Berkowicz, der Kulturbeauftragte des Stadtpräsidenten: „Krakau änderte sein Gesicht erheblich in den letzten Jahren. Aus einer Stadt, die man eher mit Konservatismus assoziierte und mit Geschichte gleichsetzte, wird es nun zu einer dynamischen und modernen Stadt. “23 Die angeführten Meinungen veranschaulichen deutlich, dass der Bau des MOCAK in Zabłocie mit zwei Fragenkomplexen zusammenhing: mit der Diskussion über die Rolle der Geschichte in der Entwicklung und Modernisierung Krakaus sowie mit der Frage des alten und des neuen Stadtzentrums.

Das Etikett der ,geistigen Hauptstadt“ haftete Krakau auch nach der Wiedererlangung der Souveränität durch Polen (1918) an. Die Stadt wurde nach wie vor als das wertvollste nationale lieu de memoire betrachtet. Krakau sollte - so die damals verbreitete Meinung - nicht zuletzt als touristischer Knotenpunkt seinen historischen Charakter wahren. ${ }^{24}$ Aus diesen Gründen stieß der Bau der Versicherungsgesellschaft Feniks am Hauptmarktplatz schon bei der Bewilligung der Baupläne auf zahlreiche Schwierigkeiten, und die modernistische Fassade des Gebäudes, das 1932 fertiggestellt wurde, auf gewaltige Kritik. ${ }^{25}$ Abb. 2 In Fortsetzung der Tradition von feierlichen und patriotisch geprägten Bestattungen bekannter Polen wurden 1929 die Asche des romantischen Dichters Juliusz Słowacki in der Kathedrale auf dem Wawel beigesetzt und sechs Jahre später - in einer speziell für diesen Zweck eingerichteten Krypta der Kirche - der Leichnam des Marschalls Józef Piłsudski. ${ }^{26}$ Geschichte bestimmte auch nach dem zweiten Weltkrieg die Wahrnehmung der

22 Hajok, Handzlik (s. Anm. 3), S. 3.

23 Zmieniamy oblicze Krakowa na nowocześniejsze (z Filipem Berkowiczem rozmawia Ryszard Kozik). In: Targoń, Kozik (s. Anm. 13), S. 5.

24 Kozińska-Witt (s. Anm. 10), S. 137-148.

25 Rafał Ochȩduszko: Projekty Adolfa Szyszko-Bohusza dla Domu Towarzystwa Ubezpieczeniowego „Feniks”w Krakowie. In: Modus. Prace z Historii Sztuki 8/9, 2009, S. 199-252.

26 Patrice M. Dabrowski: „Equal to the Kings”? Viewing Wawel Burials of the Interwar Period. In: Centropa 12, no 1, 2012, S. 4-19. 
Stadt mit. Das Begräbnis des Präsidenten Lech Kaczyński (2010), der neben dem Marschall Piłsudski bestattet wurde, zeigte vor Kurzem, dass die nationale Dimension der Ruhmeshalle auf dem Wawel nichts von ihrer symbolischen Kraft eingebüßt hatte. Die Fassade des bereits genannten Feniks-Gebäudes wurde von den Deutschen im



3: Krakau, Marktplatz mit dem Feniks-Gebäude im Umbau, 1942 (Foto Archiwum Pałstwowe Kraków).

\section{Zweiten Weltkrieg mit klassi-}

schen Pilastern verunstaltet (1941-1942). ^ Abb. 3 In der Nachkriegszeit wurden diese Zusätze nicht entfernt, ${ }^{27}$ mehr noch, heute weiß kaum jemand mehr, dass sie in der Zeit der NS-Besatzung entstanden sind. Was gilt, ist die Anpassung der Fassade an die historische Architektur des Hauptmarktplatzes. Diese Tatsachen beweisen, dass es nach wie vor die nationale Vergangenheit ist, die den Charakter Krakaus entscheidend determiniert. Auf die Zukunft gerichtete Projekte haben kaum eine Chance auf die Realisierung, besonders wenn sie moderne Architektur in dem historischen Gewebe der Stadt vorsehen.

In Krakau bildete sich auch kein anderes städtebauliches Zentrum außer dem mittelalterlichen, an dessen Rand, anstelle der alten Stadtmauer, im 19. Jahrhundert ein Park (Planty) und ein Straßenring mit zahlreichen Monumentalbauten angelegt wurden. Diese Gebäude haben bis heute ihre Funktion als bedeutende Verwaltungsstandorte beibehalten. Im historischen Stadtzentrum haben der Magistrat, das Rektorat der Jagiellonen-Universität und die meisten Banken wie auch das Woiwodschaftsamt ihren Sitz.

Die Revitalisierung von Zabłocie, mit der die Einrichtung von zwei musealen Institutionen und einer Hochschule in diesem Stadtviertel einherging, war also ein Versuch zur Bildung eines neuen Stadtzentrums. Zudem war das rechte Weichselufer stets symbolisch unterbewertet. Das alte Podgórze wollte sich mehr Prestige verschaffen, indem die Stadt eine eigene „Marienkirche“ errichtete, und

27 Rafał Ochȩduszko: Hitlerowska przebudowa Gmachu Towarzystwa Ubezpieczeniowego „Feniks” w Krakowie. In: Dariusz Nowacki (Hg.): Mecenat artystyczny a oblicze miasta, Kraków 2008, S. 303-316. 


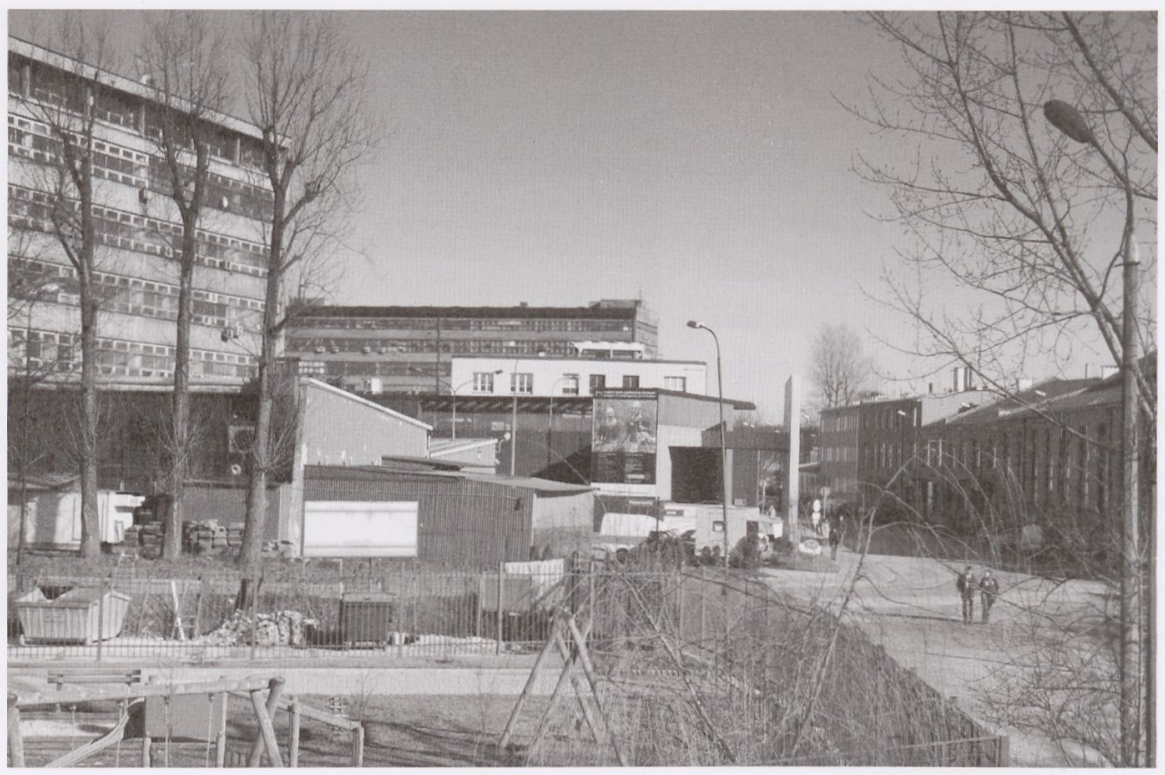

4: Krakau-Zabłocie: die Umgebung von MOCAK (das Museumsgebäude in der Mitte).

nun beherbergt das neue Zabłocie eine Hochschule, deren Name Krakowska Akademia [Krakauer Akademie] - zufällig? - die Umkehrung des altpolnischen Namens der Jagiellonen-Universität, d.h. Akademia Krakowska, ist. Postindustriale Gebiete sind nur schwach mit dem historischen Gedächtnis gesättigt. Daher lassen sie sich einfach zu einer Palimpsest-Struktur verwandeln, in der das stellenweise noch durch mittelalterliche Landstraßen vorbestimmte Straßennetz mit industrieller Architektur der Fabrikhallen und Stadtrandhäusern verschmilzt. Auf einem solchen Gebiet kommt es selten zu einem Konflikt und Kampf der Symbole. ${ }^{28}$ Ein gutes Beispiel hierfür ist Schindlers Fabrik. Das erhaltene Verwaltungsgebäude, in dem das Historische Museum untergebracht ist, erstreckt sich an der LipowaStraße entlang. $\pi$ Tafel 10 Die MOCAK-Gebäude befinden sich hinter ihm, tiefer im Grundstück. $\pi$ Tafel 11 Claudio Nardi sagte sogar, dass das Museum von der Seite der Straße unsichtbar sei, wo es lediglich durch eine gewaltige Wand mit der Inschrift MOCAK angekündigt wird. ${ }^{29}$ Somit bleiben hier die Geschichte und die Modernität in einem dialektischen Gleichgewicht. Das Museum für Gegenwartskunst versteckt sich hinter dem Historischen Museum und das Ensemble wurde in die Landschaft der Überreste anderer Fabriken und der Umrisse neuer Apartmenthäuser eingeschmolzen. $\rtimes$ Abb. 4 Das MOCAK erhebt sich keinesfalls

28 Elżbieta Rybicka: Pamięć i miasto. Palimpsest vs. pole walki. In: Teksty Drugie 5 (131), 2011, S. 201-211.

29 Muzeum jako wirus. Rozmowa z Claudio Nardim - twórça projektu MOCAK. In: Tagoń, Kozik (s. Anm. 13), S. 9. 


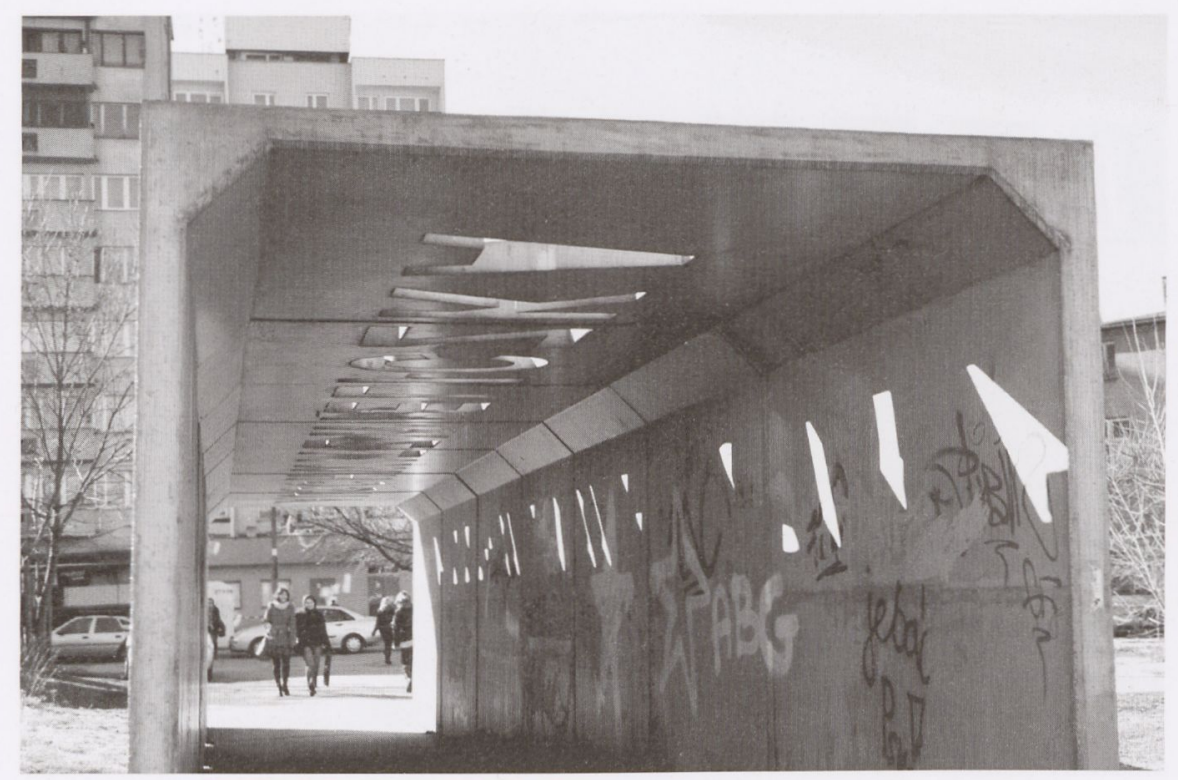

5: Mirosław Bałka, AUSCHWITZWIELICZKA, 2009.

dominierend über die übrigen Gebäude von Zabłocie. Bezieht sich denn Schindlers Fabrik überhaupt unmittelbar auf die Geschichte? Von der Seite der zentralen Teile des Stadtviertels führt der Weg nach Zabłocie unter einem Bahndamm, vor den eine Skulptur von Mirosław Bałka gestellt wurde. 入 Abb. 5 Sie entstand 2009 für das Krakauer Festival der Visuellen Künste ArtBoom und ist ein Tunnel mit der Inschrift „AUSCHWITZWIELICZKA“. Das Werk wurde durch Angebote von Tourismus-Agenturen inspiriert, die mit einem Schlag nebeneinander für die touristischen Highlights wie das Salzbergwerk in Wieliczka und das KZ-Lager in Auschwitz werben. Elektrische Touristen-Miniwagen, die auf den Straßen Krakaus zahlreich zu sehen sind, verlocken die Gäste mit ihren Werbesprüchen ebenfalls, die Route „Old Town - Kazimierz - Schindler’s Factory“ einzuschlagen. ^ Abb. 6 Die historische Dimension von Zabłocie scheint also ein touristisches Konstrukt zu sein, das von Spielbergs Film aufgetrieben wird, und weniger eine Folge seines Wertes als Gedenkstätte. Die erste Ehrentafel für Schindler wurde erst 2004 am Gebäude in der Lipowa-Straße angebracht. ${ }^{30}$ Ein solcher Ort eignet sich sehr gut für ein neues Zentrum: Fehlende größere historische Belastung lässt das Stadtviertel frei gestalten, die Erinnerung an historische Ereignisse ist dort eher ein Simulacrum als Wirklichkeit, und die Gentrifizierungs- und Modernisierungsprozesse stoßen auf keine Hindernisse. 


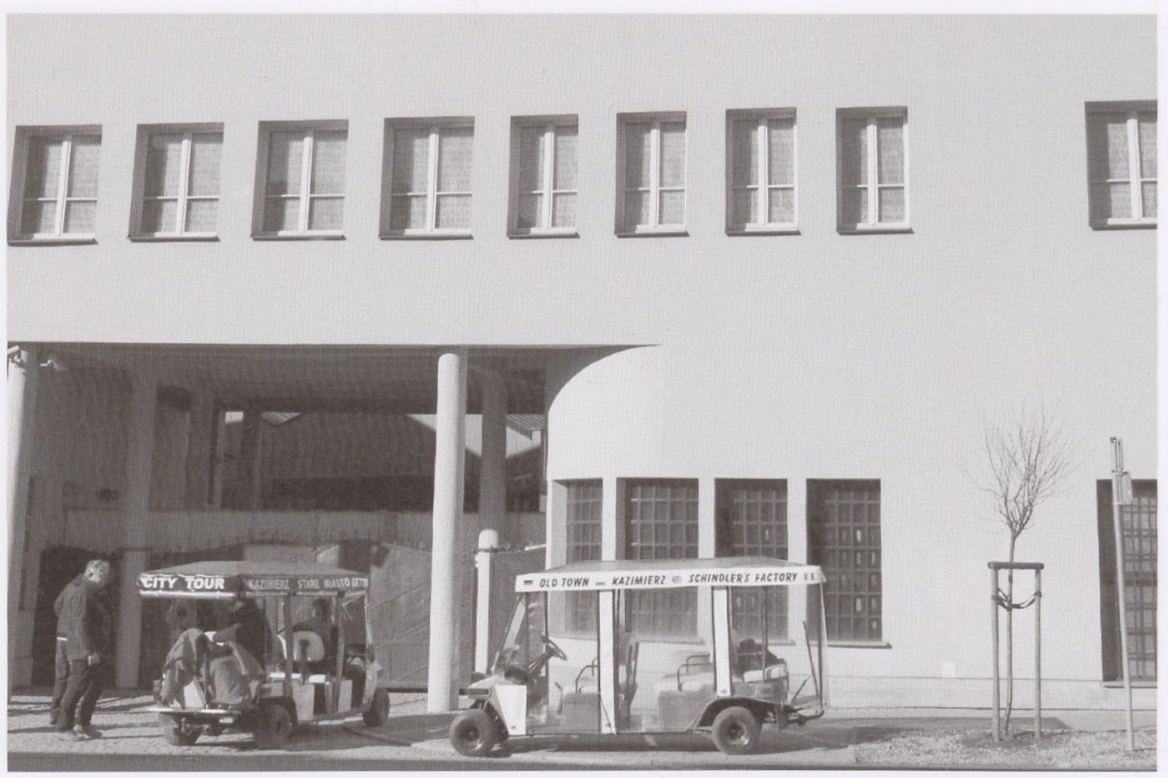

6: Elektrische Touristen-Miniwagen vor Schindlers Fabrik.

MOCAK-Direktorin Anna Maria Potocka sagte auch: „Wir legen Wert [...] auf eine harmonische Entwicklung des Stadtviertels. “31 Alles deutet darauf hin, dass der Wandel auf dem Gebiet von Zabłocie natürlich vonstatten geht: Neues verschmilzt mit Altem, die Nachbarschaft des Holocausts mit der kritischen Kunst sorgt nicht für Spannungen, und Luxus-Apartments schießen neben einer Hochschule aus dem Boden. Doch Roland Barthes hat den zeitgenössischen Menschen eine misstrauische Haltung gegenüber dem „Natürlichen“ beigebracht: Hinter dem „Natürlichen“ stecke nämlich meistens der Mythos. ${ }^{32}$ Im Falle von Zabłocie hat man auch mit einem Mythos zu tun. Es ist der Mythos einer harmonischen Entwicklung, d.h. eines Fortschritts, der dadurch umsetzbar sei, dass das neue Zentrum Krakaus ans rechte Weichselufer verlegt wird - in einen Raum, wo der für Krakau so typische Druck der großen Geschichte keine Vorhaben verhindert. 